\title{
Antibacterial prophylaxis of surgical site infections in oral surgery: Not only and not always systemic antibiotics
}

\author{
Roberto Pippi
}

Antibiotic prophylaxis (AP) is supposed to play a very important role in dental practice above all in oral surgery, in order to prevent treatment related infections, which can develop locally in the surgical wound, the so called surgical site infections (SSI), as well as at a distance from the surgical wound, the so called distant site infections (DSI).

The need of a prophylactic protocol is related to the fact that the operative field and the surgical area come unavoidably into contact with microorganisms of the rich normal bacterial flora of the oral environment as well as with those microorganisms responsible of surgical site preoperative infections.

However, since the risk of infection is not so high for all kind of surgeries, a routine use of antibiotics for prophylaxis is not justified. On the other hand, if the risk of infections is low, AP is not effective and it is also too expensive [1]. There is a lack of evidence based guidelines in the international scientific literature about AP; otherwise it is not possible to verify if infections occur or not without antibiotic prophylaxis in clinical conditions in which antibiotics are worldwide supposed highly indicated.

The wide use of antibiotics all over the world has produced two important negative effects: selection and quick development of antibiotic resistant bacterial strains; increase of allergic reactions to antibiotics. A rational use of AP is therefore necessary, based on patient local and systemic conditions as well as on the different kind of surgery.

Four different clinical situations should be considered: healthy subjects undergoing surgical procedures with a

Roberto Pippi

Affiliations: Sapienza University of Rome.

Corresponding Author: Roberto Pippi, Sapienza University of Rome; Email: roberto. pippi@uniroma1.it

Received: 14 July 2015

Published: 07 October 2015 high risk of infective complications; surgical procedures in which a secondary infection should have a high risk to compromise the final outcome, as in complex regenerative procedures or in procedures in which heterologous materials are used; patient local conditions, far from the surgical area, which predispose to bacterial colonization (DSI); patients with immune-compromised defences.

More or less validated antibiotic protocols exist from international associations with slight or no differences among them, for DSI prevention, but no evidence based indications exist for SSI. Nevertheless SSI prophylaxis should be always achieved in oral surgery not only and not always by systemic antibiotics but certainly always by means of local measures which are those applied before, during and after surgery, to reduce the number of microorganisms of the normal bacterial flora and to avoid exogenous bacteria reach the surgical site by means of surgeons or/and surgical instrumentation (Table 1).

Most of oral surgical procedures, short in time and less invasive, only need a clean operative field; this is the case of tooth extractions, endodontic and minor preprosthetic surgery, biopsies and others. In these cases, sterile instrumentation, single use gloves and masks, clean overalls, protective glasses and headgears are needed. A sterile field, on the contrary, should be used in more invasive surgeries or when heterologous materials are inserted; in these cases sterile gloves and overalls are needed, the patient should be covered by sterile sheets, and peri-oral skin disinfections should be performed.

Carefulness is required in all surgical steps, especially in soft tissue management, to avoid flap lacerations, in

Table 1: Antibacterial local measures

- use of sterile instrumentation and special devices to prevent surgical site contamination

- good surgical treatment

- pre-surgical treatment of acute local infections

- pre-surgical calculus removal and perioperative plaque control

- post-surgical follow-up 
providing copious saline irrigation, during and after bone drilling, to avoid overheating and to remove debris, and finally in suturing, to achieve surgical flap perfect stabilization, without any tension, in order to avoid wound dehiscence. Suturing is often underestimated but it is an important step in avoiding SSI, from the choice of suture material, which should be as less phlogogenic as possible to induce little inflammation, to correct positioning of knots, that should be on one side of the incision line to avoid plaque accumulation just over the incision line. Suture removal is important as well, to avoid microorganisms are carried inside the tissues during thread removal. Therefore, the thread must be cut just near the tissue surface so that only a minimal portion of the contaminated suture material passes through the soft tissues during its removal. On the other side, when a second intention healing is going to take place, a surgical dressing may be applied for wound protection by infections and traumas. Finally, operative quickness is desired to make microbial contamination as little as possible.

If an acute local infection is already present in the surgical area, the risk of microbial spreading due to surgical instrumentation is very high. Every acute infection, such as pericoronitis, abscess or sinusitis, should be therefore resolved with local and systemic therapies before surgery is scheduled.

Pre-surgical calculus removal is a good rule to avoid calculus fragments may accidentally be displaced into the surgical field and they may interfere with the wound healing. Calculus should be removed at least three days before surgery, so that gingival tissues are healthy and can be easily managed during surgery. Just before surgery a $0.20 \%$ chlorhexidine mouth wash should be used for rinsing one minute the mouth in order to reduce the microbial charge of the oral environment. Postoperatively, traumas must be avoided on surgical area and wound has to be perfectly and steadily cleaned. In case of flapless (nonsurgical) extractions, blood-clot trouble must be avoided during the first 6-8 hours after extraction: no rinsing, no spitting, no chewing is permitted and only a cold and liquid diet should be followed. In the same time interval, oral hygiene techniques should be avoided and $0.20 \%$ chlorhexidine solutions, like gel, vaporizer, mouth wash, should be used. After 8 hours, it is possible to gently brush the teeth with a soft bristles tooth-brush, but not in the extraction area. Only 2-3 days after the extraction, the common devices and techniques for oral hygiene can be carefully restarted to use. During the early healing process (at least 5-7 days) smoke and alcohol intake as well as hard food chewing on extraction area should be avoided. In case of surgical extractions, a cold dressing is advisable to be applied extra-orally in the surgically treated area, for 2-3 hours intermittently. Cold applying is controversial but it seems to allow the postoperative oedema be less extensive so that the risk of infection due to vascular stasis is reduced and the patient can more easily accomplish his/her daily oral hygiene measures. Surgical wound cleaning should be performed with a cotton swab soaked in 12 vol. $\mathrm{H}_{2} \mathrm{O}_{2}, 50 \%$ water down. Furthermore, smoking and hard food chewing should be avoided until suture will be removed, after $5^{-7}$ days. If a dehiscence occurs in the surgical wound above the residual socket, irrigation should be performed two-times a day with a syringe to clean the bone cavity from food debris. A second intention healing will therefore occur. In case of demolishing, reconstructive, regenerative, implant or plastic surgeries in masticatory areas, smoke and alcohol intake as well as chewing in those areas should be forbidden for at least two weeks, anyway until sutures and possible surgical dressing will be removed. Only a liquid diet should be followed in that period and a careful hygiene of surgical wound with chlorhexidine and $\mathrm{H}_{2} \mathrm{O}_{2}$ should be maintained. After the first two weeks, a soft diet should be followed and if obturators or protective/ cover plates are present, they should be removed and accurately cleaned after each meal.

A precise and strict follow-up program should be started in order to early detect any problem that may interfere with the healing process. On each appointment, the surgical area should be carefully examined and cleaned and specific directions for improving oral hygiene and wound healing should be given to the patients and each time reinforced.

As to systemic treatment, in order to be safe and useful, AP of SSI should follow the following five basic principles:

(1) only procedures that have high risk of infection need AP

(2) an adequate antibiotic should be chosen

(3) a high dose of antibiotic should be used

(4) the time of administration should be correct

(5) antibiotic activity should be as short as possible

(1) The high risk of infection can be related to general or/and local patient's predisposing conditions. Among general ones, the following are included: immunecompromising or organic debilitation due to old age or co-morbidities which may justify an overall reduction of patient immunity defences, inability to provide to daily oral hygiene, impossibility to undergo post-surgical follow-up. As concern local predisposing conditions, they are related to the type, site, extension and duration of surgery as well as to pre-existing infections. Particular cases are those in which heterologous materials are implanted (implant and regenerative surgeries), openfractures are treated or the maxillary sinus is involved with membrane opening.

(2) Antibiotic choice is based on three basic aspects: selective effectiveness, i.e. effectiveness against bacteria responsible of infections which are intended to be prevented, low toxicity and bactericidal activity. Penicillins satisfy all these requirements. In case of allergy to penicillins, clindamycin or cephalosporins can be used, although with the latter, hypersensitivity crossreactions with penicillins can occur. If a trans-cutaneous intervention has to be performed, first generation 
cephalosporins should be preferred because of their better effectiveness against staphylococci. Macrolides can also used as a last choice.

(3) The recommended dose is twice the therapeutic one, so that plasmatic and tissue concentrations are double too.

(4) In order to allow the antibiotic blood concentration is highest when the surgery is going to start, administration should be performed 1 hour before, if orally, and half an hour before, if parenterally. No further doses are usually necessary for oral surgeries since they do not last more than antibiotic blood half time. However, a second dose can be administrated if the procedure lasts more than two hours, intraoperatively or postoperatively, but before the patient is discharged.

(5) Antibiotic prophylaxis should therefore last as less as possible and only in few very complex or particularly long lasting cases it could be continued with further doses after 6-12 hours, but always not over 24 hours. Actually, in not complex procedures, short duration AP seems to be as effective as a long-term AP. Antibiotic prophylaxis should also be started within 1 hour and not over 2 hours from the end of the surgery and it should be continued for some days, if during surgery an accident occurs with a high risk of infection. This is the case of oroantral communication, wide bone fractures involving the alveolar process, the maxillary tuberosity or the mandible, hematomas and subcutaneous emphysema.

As a general rule, according to Altemeier classification [2], elective oral surgeries belong to the clean surgeries whose postoperative infection rate ranges from $1-4 \%$ and for which AP is therefore not indicated in healthy patients. Actually, no statistically significant relationship has never been found between the incidence of postoperative infection in most oral surgical procedures and AP [3]. Nevertheless recent reviews showed evidences that AP reduces the risk of postoperative infection in two common surgical procedures, such as third molar extraction [4] and dental implant placement in ordinary conditions [5], although authors were not able to clarify whether or not postoperative antibiotics are useful $[4,5]$. However, those reviews $[4,5]$ did not take into account the different complexity and difficulty of surgeries as well as the different surgeon's experience and all other possible variables which may increase the risk of SSI. Actually local and general as well as environmental and climatic conditions may increase such a risk, so that guidelines are not generalizable to all cases within the same type of surgical procedure. Anyway, the greater the risk of infection the closer the follow-up sessions should be, especially if AP was not prescribed, and an antibiotic therapy should be started as soon as a postoperative infection appears [6]. Moreover, the high number of patients which need to be treated with AP for one additional beneficial outcome, i.e. for preventing a postoperative infection in one patient, raises serious questions about the real utility of AP if compared with the risks of bacterial resistance and abnormal treatment responses. Furthermore, most oral SSI are not severe, early diagnosable by means of an adequate follow-up, and easily treatable in few days, with an appropriate antibiotic therapy. Lastly, the overall cost for antibiotic treatment of the few cases of oral SSI is very much lower than the overall cost to perform AP in all oral surgeries.

In conclusion, antibacterial prophylaxis is a set of treatment measures, including AP, under surgeon's responsibility. If local measures are always to be applied, AP administration needs a careful case selection in order to avoid indiscriminate prescription.

Keywords: Antibiotics, Antibiotic prophylaxis, Oral surgery, Surgical site infection

\section{How to cite this article}

Pippi R. Antibacterial prophylaxis of surgical site infections in oral surgery: Not only and not always systemic antibiotics. Edorium J Dent 2015;2:43-46.

\section{Article ID: 100009Do1RP2015}

\section{$* * * * * * * * *$ \\ doi:10.5348/Do1-2015-9-ED-7 \\ $* * * * * * * * *$}

\section{Author Contributions}

Roberto Pippi - Substantial contributions to conception and design, Acquisition of data, Analysis and interpretation of data, Drafting the article, Revising it critically for important intellectual content, Final approval of the version to be published

\section{Guarantor}

The corresponding author is the guarantor of submission.

\section{Conflict of Interest}

Authors declare no conflict of interest.

\section{Copyright}

(C) 2015 Roberto Pippi. This article is distributed under the terms of Creative Commons Attribution License which permits unrestricted use, distribution and reproduction in any medium provided the original author(s) and original publisher are properly credited. Please see the copyright policy on the journal website for more information.

\section{REFERENCES}

1. Lockhart PB, Blizzard J, Maslow AL, Brennan MT, Sasser H, Carew J. Drug cost implications for antibiotic prophylaxis for dental procedures. Oral Surg Oral 
Med Oral Pathol Oral Radiol 2013 Mar;115(3):34553 .

2. Altemeier WA. Surgical infections: incisional Wounds. In: Bennett JV, Brachman PS, eds. Hospital infections. Boston: Little, Brown and Co; 1979. p. 87306.

3. Powell CA, Mealey BL, Deas DE, McDonnell HT, Moritz AJ. Post-surgical infections: prevalence associated with various periodontal surgical procedures. J Periodontol 2005 Mar;76(3):329-33.

4. Lodi G, Figini L, Sardella A, Carrassi A, Del Fabbro M, Furness S. Antibiotics to prevent complications following tooth extractions. Cochrane Database Syst Rev 2012 Nov 14;11:CDo03811.

5. Esposito M, Grusovin MG, Worthington HV. Interventions for replacing missing teeth: antibiotics at dental implant placement to prevent complications. Cochrane Database Syst Rev 2013 Jul 31;7:CDo04152.
6. Pippi R. Antibiotic prophylaxis: reasoned choice and not casual use. Ann Stomatol (Roma) 2011 Mar;2(34):1-2.

\section{SUGGESTED READING}

- $\quad$ Laskin DM. Should prophylactic antibiotics be used for patients having removal of erupted teeth? Oral MaxillofacSurgClin N Am 2011;23:537-539.

- Lopes DR, Peres MPSM, Levin AS. randomized study of surgical prophylaxis in immuno-compromised hosts. j Dent Res 2011;90(2):225-229.

- Termine N, Panzarella V, Ciavarella D, Lo Muzio L, D’Angelo M, Sardella A, Compilato D, Campisi G. Antibiotic prophylaxis in dentistry and oral surgery: use and misuse. Int Dent J 2009;59(5):262-270.
Access full text article on other devices

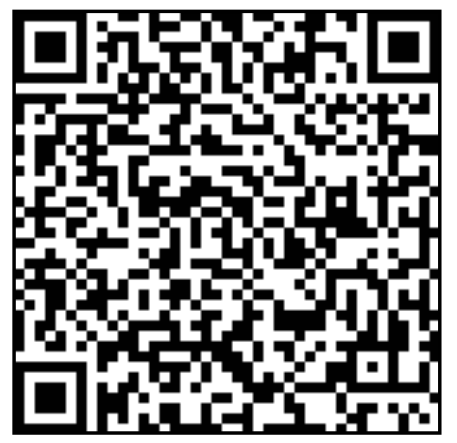

Access PDF of article on other devices

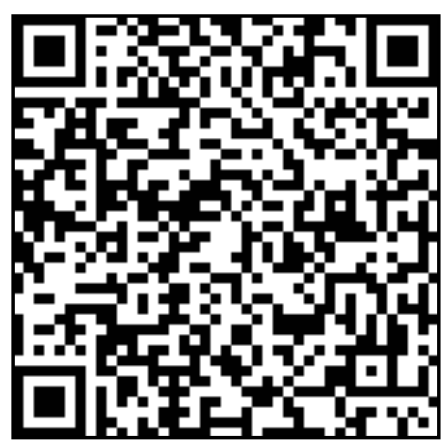

